\title{
English Staffordshire Terrier
}

National Cancer Institute

\section{Source}

National Cancer Institute. English Staffordshire Terrier. NCI Thesaurus. Code C53735.

The Eng lish Staffordshire Bull Terrier is a powerful and muscular dog, with a broad head and very strong jaws. It has a short, soft coat that comes in black, blue, fawn or brindle, often with white markings. The ears are either rose or half-pricked. Height: 13-16 inches (33-41 cm.) Weight: 23-38 pounds (10-17 kg.) 